\title{
OPTIMIZED THERMAL SIMULATION MODEL OF MULTILAYER PRINTED CIRCUIT BOARD
}

Very thin multi-layers of $\mathrm{Cu}$ and epoxy compound in PCB (printed circuit board) make difficult to design computational meshes in finite element method simulation programs. Number of degrees of freedom in simulated system rapidly increases - it significantly extends simulation computational time. In this paper we propose substitution of the PCB in axial and radial direction with the composite structure with equivalent physical parameters of multilayer PCB.

Keywords: multilayer printed circuit board, thermal modeling, approximation, simulation

\section{Introduction}

Considering high degree of geometrical complexity of electronic products which are being developed, it is quite important to focus on the preconstruction phases within the development process. It is well known, that efficiency is the main indicator that defines the qualities of any electronic system. Efficiency is directly dependent on the temperature, because any unwanted temperature rise in electronic systems is related to the existance of undesirable power losses. When complex systems, like integrated circuits, i.e. switched mode power supplies SMPS are considered the efficiency is the most important parameter [1-3].

Nowadays, the market offers various rapid prototyping software, thus enabling the researchers fast identification of unwanted processes and consequent system optimization. When talking about SMPS it is valuable to perform identification of the thermal performance. The simulation model is very valuable because fast and very accurate results can be achieved [4-6]. Consequently, optimization processes are much more effective to be done with the use of good thermal simulation model [7, 8]. On the other side, there are some challenges which are related to the complexity of such systems. It is well known that any SMPS consists of huge number of active and passive components, which might be modeled in order to have very good simulation results. At the same time, printed circuit board PCB has to be modeled too, because it is a part of each SMPS. It is known, that multilayer PCB are being used in almost every hi-tec SMPS design. Multilayer PCBs are composed of several interleaved layers of copper, FR4 material and other mask materials. These layers represent a very thin structure and again, when precise simulation model is required, then each layer must be considered.

In this paper the optimized thermal simulation model of multilayer PCB is shown, whereby we have focused on factors, which are affecting thermal conductivity of multilayer PCB. Modeling of the PCB in the way that FR4 material for whole subdomain of $\mathrm{PCB}$ is assigned is not correct. Thin copper layers which are placed on the TOP and BOTTOM side of PCB, and even inside of $\mathrm{PCB}$ subdomain, have high impact on thermal distribution on the surface of $\mathrm{PCB}$ as well as inside of this subdomain.

The main problem of multilayer PCB is thin copper layer (35um - 100um) in combination with epoxy layers. This thin geometry of copper in combination with the other neighboring epoxy layers produces a high number of mesh elements what in final makes the simulation model more complex. Then the simulation time of solution is much longer. We propose to replace physical properties of multilayer PCB by thermal conductivity tensor in radial and axial part, which will have the same properties as the mentioned multilayer PCB structure.

\section{Principle of PCB thermal model optimization}

In this paper, the basic study of the behavior of multilayer PCB and consequently possibilities how to simplify such geometry

\footnotetext{
* ${ }^{1}$ Michal Frivaldsky, ${ }^{1}$ Pavol Spanik, ${ }^{1}$ Jan Morgos, ${ }^{2}$ Norbert Glapa

${ }^{1}$ Department of Mechatronics and Electronics, Faculty of Electrical Engineering, University of Zilina, Slovakia

${ }^{2}$ Panasonic Industrial Devices, GmbH, Luneburg, Germany

E-mail: michal.frivaldsky@ef.uniza.sk
} 


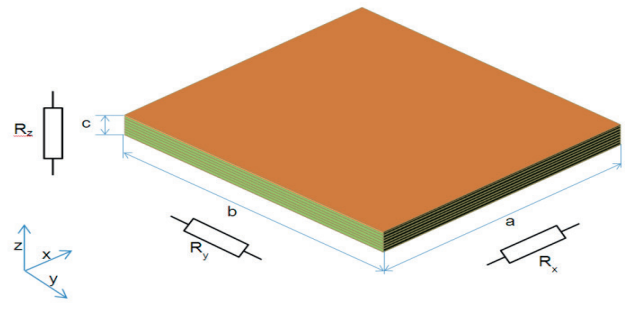

Figure 1 Simple model of multilayer PCB board

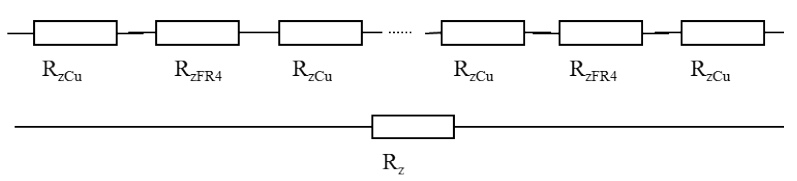

Figure 2 Thermal resistance of $P C B$ in z-direction

are given. Multilayer PCB is replaced by geometrically and physically similar composite whose physical properties will be accurate and sufficient for thermal simulations in COMSOL environment.

Considering axial and radial direction of PCB structure, it has different conditions for heat transfer within its volume. The fact is given by the structural compound of the PCB, while most used materials are FR4 and $\mathrm{Cu}$ mutually interleaved. These processes can be related to the different values of the thermal resistances in $x, y, z$ directions in $3 \mathrm{D}$ space according to Figure 1 .

In Figure 1 it is seen, that interleaved copper layers and epoxy compound layers form sandwich structure of the PCB. Thermal resistances of the entire board in $\mathrm{x}$ and $\mathrm{y}$ directions differ from thermal resistance in $\mathrm{z}$ direction.

Cross-sectional thermal resistance in $\mathrm{z}$ axis is derived and based on the sandwich structure in Figure 2 as follows: it consists of separate layer thermal resistances connected in series, whereby the number of FR4 material and $\mathrm{Cu}$ material is based on the number of layers of PCB.

According to Figure 2 the thermal resistance of $\mathrm{PCB}$ structure in $\mathrm{z}$-axis direction is given by the Equation (1) as follows:

$R_{z}=\sum_{k=1}^{n} R_{z C u}(n)+\sum_{k=1}^{n} R_{z F R 4}(m)=n \cdot R_{z C u}+m \cdot R_{z F R 4}$

where:

$R_{z C u}$ - thermal resistance of copper

$R_{z F R 4}$ - epoxy compound FR4 type,

$n$ - number of copper layers,

$m$ - number of epoxy layers.

Thermal resistance (and therefore thermal conductivity) in „“ $x^{\prime}$ and „y“ directions are representing parallel connection of resistances of several PCB layers.

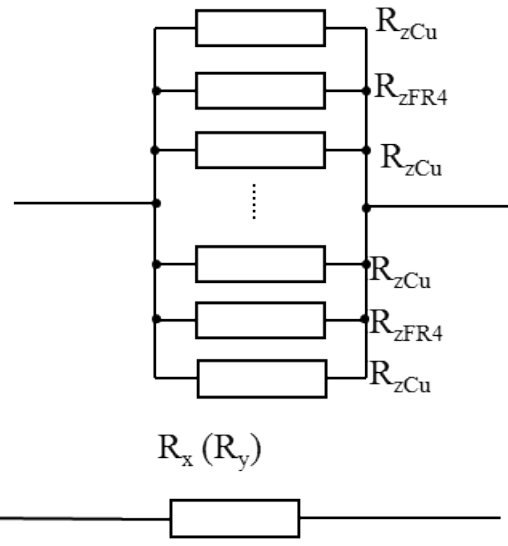

Figure 3 Thermal resistance of $P C B$ in $x, y$ direction

According to Figure 3 the thermal resistance of PCB structure in $x, y$ - axes is inverse to the Equation (1) and thus based on the Equation (2) it is possible to determine its values as follows:

$$
\begin{aligned}
R_{x}\left(R_{y}\right) & =\frac{1}{R_{z}}=\sum_{k=1}^{n} \frac{1}{R_{z C u}(n)}+\sum_{k=1}^{m} \frac{1}{R_{z F R 4}(m)_{z}}= \\
& =n \cdot \frac{1}{R_{z C u}}+m \cdot \frac{1}{R_{z F R 4}}
\end{aligned}
$$

Small layers of PCB (Figure 1) represent interconnected system of very thin blocks with the same surface and different thickness of copper or epoxy compound. Thermal resistance of block in 3D space for $x, y, z$ directions can then be simplified into the next Equation (3):

$$
\begin{aligned}
& R_{x}=\frac{\Delta x}{k_{x} \cdot A_{y z}} \\
& R_{y}=\frac{\Delta y}{k_{y} \cdot A_{x z}} \\
& R_{z}=\frac{\Delta z}{k_{z} \cdot A_{x y}}
\end{aligned}
$$

where:

$\Delta x \quad$ - thickness of block in $\mathrm{x}$ direction,

$A_{y z} \quad$ - area which thermal flow runs through in perpendicular direction,

$\Delta y \quad$ - thickness of block in y direction,

$A_{x z} \quad$ - area which thermal flow runs through in perpendicular direction,

$\Delta z \quad$ - thickness of block in $\mathrm{z}$ direction,

$A_{x y} \quad$ - area which thermal flow runs through in perpendicular direction,

$k_{x}, k_{y}, k_{z}$ - thermal conductivity of material in the given direction.

For thermal simulation model of the PCB it is necessary to derive thermal conductivities in each direction. In principle the thermal conductivity is the inverse value of the thermal resistance, thus based on the Equation (3) it is possible to derive equations 


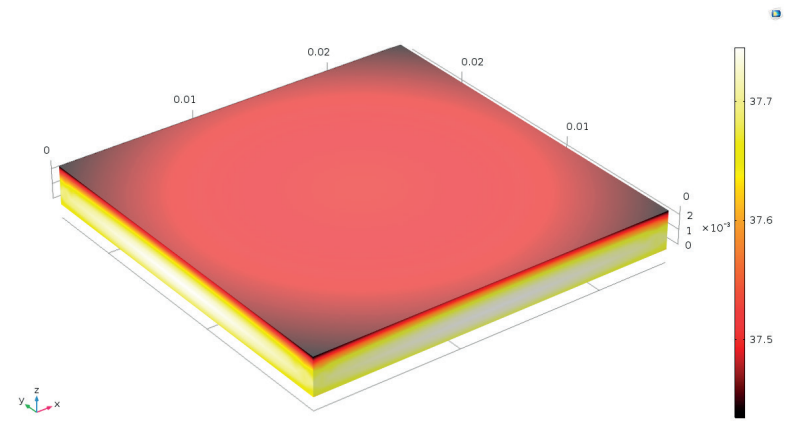

Figure 4 Surface temperature distribution in simulated model of PCB $8 x C u+7 x F R 4$

for the computation of thermal conductivities of multilayer PCB in the following Equation (4):

$$
\begin{aligned}
k_{z} & =\frac{n \cdot w_{C u}+m \cdot w_{F R 4}}{\frac{n \cdot w_{C u}}{k_{c u}}+\frac{m \cdot w_{F R 4}}{k_{F R 4}}}=\frac{w}{\frac{n \cdot w_{C u}}{k_{C u}}+\frac{m \cdot w_{F R 4}}{k_{F R 4}}} \\
k_{x} & =\frac{n \cdot k_{C u} \cdot w_{C u}+m \cdot k_{F R 4} \cdot w_{F R 4}}{n \cdot w_{C u}+m \cdot w_{F R 4}}= \\
& =\frac{n \cdot k_{C u} \cdot w_{C u}+m \cdot k_{F R 4} \cdot w_{F R 4}}{w} \\
k_{y} & =\frac{n \cdot k_{C u} \cdot w_{C u}+m \cdot k_{F R 4} \cdot w_{F R 4}}{n \cdot w_{C u}+m \cdot w_{F R 4}}= \\
& =\frac{n \cdot k_{C u} \cdot w_{C u}+m \cdot k_{F R 4} \cdot w_{F R 4}}{w}
\end{aligned}
$$

where:

$w_{C u}$ - thickness of copper layer

$w_{F R 4}$ - thickness of epoxy layer

$w$ - total thickness of PCB

$k_{C u}$ - copper thermal conductivity

$k_{F R 4}$ - epoxy layers thermal conductivity

The previous simple analysis allows using Equations (1) - (4) for derivation of the required values of thermal conductivities for any multilayer PCB. These values together with material properties are then necessary for the development of optimized thermal simulation model.

\section{Simulation results}

For the implementation purposes, we have selected geometrical dimensions of PCB of brick DC-DC converter sample $[9,10]$. Independent simulations in COMSOL environment with thermal module were accomplished with two samples of PCB with the same dimensions $24.4 \mathrm{~mm} \times 27.9 \mathrm{~mm}$. The same value of power dissipation $0.2 \mathrm{~W}$ was applied within the volume of the PCB sample. The boundary conditions for both of samples were set to the same conditions (external temperature $20{ }^{\circ} \mathrm{C}$, natural laminar flow - COMSOL setting of boundary condition when radiation is considered and natural external convection).

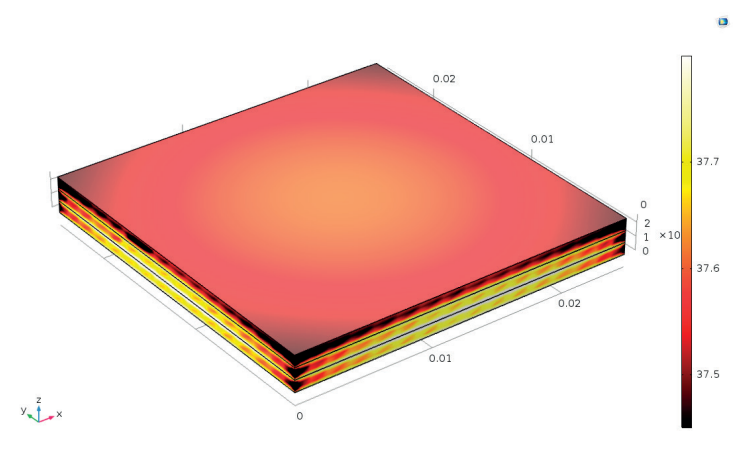

Figure 5 Surface temperature distribution in simulated composite model of $P C B$

The first model is the structured detailed sample, which contains 8 copper layers with thickness $w_{C u}=0.035 \mathrm{~mm}$, thermal conductivity $k_{\mathrm{Cu}}=400 \mathrm{~W} / \mathrm{m} . \mathrm{K}$ and 7 epoxy compound layers with thickness $w_{F R 4}=0.3 \mathrm{~mm}, k_{F R 4}=0.3 \mathrm{~W} / \mathrm{m} . \mathrm{K}$. The mesh size for this model is pretty complex, because the model consist of several thick layers. Complete mesh consists of 307632 domain elements, 113304 boundary elements and 3900 edge elements.

The second sample contained one block of composite material with thickness $2.38 \mathrm{~mm}$ and thermal conductivity as tensor with components calculated from Equation (4), $k_{x}=47.3235 \mathrm{~W} / \mathrm{m} . \mathrm{K}$, $k_{y}=47.3235 \mathrm{~W} / \mathrm{m} . \mathrm{K}, k_{z}=0.3399 \mathrm{~W} / \mathrm{m} . \mathrm{K}$. For this case the mesh consists of 92227 domain elements, 23542 boundary elements and 708 edge elements.

The graphic representation of temperature field distribution on PCB board surface is shown in Figure 4 and Figure 5. Figure 4 shows 3D graphical results of 8-layer PCB board while Figure 5 shows 3D graphical results of composite PCB board. It is seen that results of temperature distribution within the volume/area are almost identical when both models are compared. Instead of that, computation time for composite model has decreased compared to structural model from 42 seconds to 4 seconds. This result is valuable, because the decrease factor is 10 . For more complex simulation models, the save of time might be beneficial.

Figure 6 gives the detailed comparison of the internal temperature of 8-layer PCB board and composite PCB board in the z-axis cross-section of PCB in its middle point. Based on this results it is seen, that the temperature difference inside the PCB board is almost negligible, while just value of $0.02{ }^{\circ} \mathrm{C}$ is overreached. Figure 7 shows the percentage representation of relative temperature difference.

\section{Conclusion}

Contemporary construction of electronic power equipment utilizes as a mechanical and electrical base a multilayer PCB board. Single semiconductor elements and integrated circuits are built-in the board. It is suitable to reduce the number of thin and large planes by creation of geometric model of electronic 


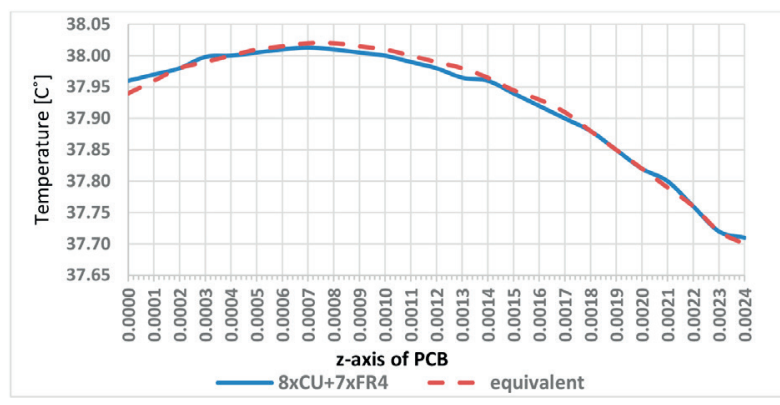

Figure 6 Comparison of internal temperatures in the middle of PCB board $8 x C u+7 x F R 4$ and in composite model

equipment. This is the case of multilayer PCB board. Authors used a composite physical model applicable for the temperature simulation needs. The equivalent model unlike a multilayer geometry significantly decreases the number of elements of computational mesh. The results of simulation confirm that the temperature fields in 8-layer PCB board and in composite PCB board differ very little. The results of simulation of PCB board samples for DC-DC converter with dimensions $24.4 \mathrm{~mm} \mathrm{x}$ $27.9 \mathrm{~mm}$ show that relative temperature deviation is $0.04 \%$ in the worst case.

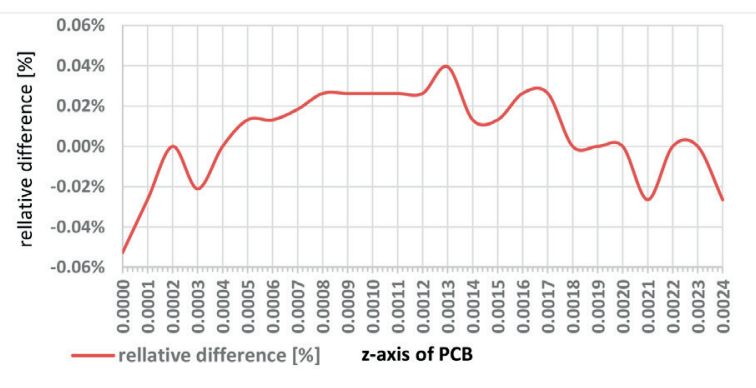

Figure 7 Relative temperature difference cross-line section plots of the $8 x C u+7 x F R 4$ and equivalent $P C B s$

\section{Acknowledgements}

The authors wish to thank for the support to the R\&D operational program Centre of excellence of power electronics systems and materials for their components. The project is funded by European Community, ERDF - European regional development fund. Authors wish also thank to national grant agency APVV for project support Nr. APVV-0396-15.

\section{References}

[1] HARGAS, L., HRIANKA, M., LAKATOS, J., KONIAR, D.: Heat Fields Modelling and Verification of Electronic Parts of Mechatronics Systems. Metalurgija (Metalurgy), 49(2), 2010.

[2] HRIANKA, M., CUNTALA, J., LAKATOS, J., HARGAS, L., KONIAR, D.: Thermal field modeling of the power electronic systems (in Slovak). Acta Mechanica Slovaca 12(3-B), 281-284, 2008.

[3] COMSOL: Multiphysics user guide.

[4] SPANIK, P., CUNTALA, J., FRIVALDSKY, M., GLAPA, N., MADLENAK, D.: Thermal Simulation of Electrochemical Double Layer Capacitor. Proceedings of the 8th International Conference ELEKTRO 2010, Slovak Republic, 62-66, 2010.

[5] SHAHIN, A., PAYMAN, A., MARTIN, J. P., PIERFEDERICI, S., MEIBODY-TABAR, F.: Approximate Novel Loss Formulae Estimation for Optimization of Power Controller of DC/DC Converter. Proceedings of 36th Annual Conference on IEEE Industrial Electronics Society (IECON 2010), USA, 373-378, 2010.

[6] SARAJCEV, I., MAJSTROVIC, M., MEDIC, I.: Calculation of Losses in Electric Power Cables as the Base for Cable Temperature Analysis. Advanced Computational Methodes in Heat Transfer VI, 27, 529 - 537, 2000.

[7] GAMIL, A., GUELDNER, H., BOEHME, F., WOLF, H.: Determine the efficiency of any power electronic device using heat transfer theory. Proceedings of the Fifth International Conference on Electrical Machines and Systems (ICEMS 2001), China, 1, 488-491, 2001.

[8] BOREKCI, S., ACAR, N. C..: An Accurate Way of Determining BJT's Switching Loss in Medium and High Voltage Applications. Proceeding of IEEE International Conference on Electronics Design, Systems and Applications (ICEDSA 2012), Malaysia, 183187, 2012.

[9] APSE-APSITIS, P., AVOTINS, A., RIBICKIS, L.: Self-Tuning Core-Less Serial Resonant DC/DC Converter for Powering Loads on Rotating Shafts. Electronics and Electrical Engineering, 19(2), 41 - 44, 2013.

[10] DOBRUCKY, B., PRAZENICA, M., KASCAK, S., KASSA, J.: HF Link LCTLC Resonant Converter with LF AC Output. Proceedings of 38th Annual Conference on IEEE Industrial Electronics Society (IECON 2012), 447-452, 2012. 